\title{
The correlation between diabetes mellitus type ii and the increased risk of alzheimer's disease: a collaborative treatment approach
}

\begin{abstract}
Diabetes Mellitus type II is among the most common and debilitating diseases in the United States. Each year, the incidence of diabetes in the U.S. is 1.4 million. ${ }^{1}$ It is estimated that by the year 2050, if current trends persists that one-third of American adult population will be diagnosed with diabetes. ${ }^{1}$ Statistics have indicated that Alzheimer's disease affects approximately one-ninth $(11 \%)$ of older Americans, ages 65 and older. ${ }^{2}$ It is further established that there will be a steady annual increase in the incidence and prevalence of $\mathrm{AD}$, as older Americans continue to live longer than 65 years. ${ }^{4}$ This paper supports the literature that links diabetes with Alzheimer's disease. It recognizes that the coexisting complications of diabetes mellitus type II is both pervasive and disheartening. Nonetheless, we have identified both physiological and pharmacological factors that are relevant to the treatment of this epidemic. In addition, we contend that further research is needed to prevent and effectively treat both diseases. An expected outcome is that sustained circumvention of the negative side effects could lead to enhanced quality of life. We conclude that based on gender and racial disparities, the most at risk populations must be identified and targeted by healthcare educators and public health officials. ${ }^{3}$ One implication is that responsible intervention will require a collaborative approach, which combines lifestyle changes and conventional medical approaches. This can result in improved clinical outcomes, patient longevity, and overall enhanced quality of life.
\end{abstract}

Keywords: CI, cognitive impairments; AD, alzheimer's disease; HDLs, highdensity lipoproteins; VLDLs, very-low-density lipoproteins; IDLs, intermediate-density lipoproteins; IAPP, islet amyloid polypeptide
Volume I Issue 4 - 2017

\author{
Kelly Stashauna,' Newton David,' Henry \\ Suzette,' Meloche Theresa M, ${ }^{2}$ Bentley \\ Ryan, ${ }^{3}$ Brown Tony $L^{2}$ \\ 'Major General Hugh G Robinson Center for Neuropsychiatry \\ Studies, USA \\ ${ }^{2}$ Faculty of Arts and Sciences, Harvard University, USA \\ ${ }^{3}$ Department of Family and Community Medicine, Western \\ Michigan University, USA
}

Correspondence: Tony L Brown, Faculty of Arts and Sciences, Harvard University, USA, Email tbrown@post.harvard.edu

Received: August 08, 2017| Published: November 24, 2017

\section{Introduction}

Diabetes Mellitus type II is among the most common and debilitating diseases in the United States. There is evidence to support a strong association between type II Diabetes and $\mathrm{AD}$, in addition to the relationship of APO E with both diseases. This is indicated in mounting evidence now suggesting that there exists a correlation between diabetes mellitus type II and Alzheimer's disease (AD)..$^{5-31}$ Diabetes type II individuals produce higher amounts of insulin compared to non-diabetics. Physiologically, the release and function of Insulin is a tightly regulated process, which enables the body to balance its metabolic needs. This hormone possesses the ability to cross the blood brain barrier and interrupt brain chemistry. When this happens, optimal brain function diminishes leading to mild cognitive impairments $(\mathrm{CI}){ }^{8,9}$ It has been described that reduced insulin action or insulin deficiency could contribute to cognitive deficiencies. ${ }^{30-33}$ Betaamyloids $(A \beta)$, are proteins unique to both Diabetes and Alzheimer's disease. Ultimately, multiple amyloid proteins build up in the brain because of excess cerebral insulin leading to Alzheimer's disease. Although $A \beta$ expression has been detected in extrabrain regions, its effects on these peripheral tissues are not yet fully understood. Studies in rodents suggest a role for $A \beta$ in regulating the ability of peripheral tissues to respond to insulin. ${ }^{8-37}$

Given that diabetes mellitus type II and AD are complex diseases, it is evident that there are several contributing factors that may increase one's likelihood of developing this disease. ${ }^{10}$ In 2014, Alzheimer's disease was recorded as the fifth leading cause of death in the U.S. and Diabetes Mellitus was ranked the sixth leading cause of death. ${ }^{11}$ The number of Alzheimer's disease related deaths totaled 93, 541 and diabetes mellitus related deaths reached a staggering total of $76,488 .{ }^{1-4}$ Of particular note is the fact that the incidence of diabetes is rapidly rising amongst individuals less than twenty years of age. According to this statistic approximately 208,000 American youths are diagnosed with diabetes. ${ }^{1}$ These findings are difficult to ignore and compel healthcare professionals to implement strategies that will support healthy lifestyle changes. This approach can decrease the incidence of both diseases while improving overall patient outcomes and preventing complications to those already diagnosed.

\section{Diabetes and american society}

According to the American Diabetes Association, the prevalence of diabetes in 2012 was 29.1 million Americans or 9.3\% of the population. Among the 29.1 million, 21 million were diagnosed and 8.1 million were never diagnosed. ${ }^{1}$ The prevalence in seniors, age 65 and older, was 11.8 million or $25.9 \%$ both who were diagnosed and undiagnosed. ${ }^{1}$ Each year, the incidence of diabetes in the U.S. is 1.4 million. It is estimated that by the year 2050, should current trends persists, one-third of American adult population will be diagnosed with diabetes. ${ }^{1}$ There is evidence to support the notion that racial and ethnic disparities exist among those with diabetes and Alzheimer's disease. ${ }^{1}$ In Figure 1, statistics have shown that $15.9 \%$ of American Indians, $13.2 \%$ non-Hispanic blacks and $12.8 \%$ Hispanics are currently diagnosed with diabetes. In addition the reported numbers of infected individuals are decreased to $9.0 \%$ of Asian Americans 
and $7.6 \%$ in non-Hispanic white Americans. The high incidence of diabetes type II among these groups can be attributed to the following predisposing factors; 1) increased accessibility to high calorie refined foods, 2) obesity, and 3) a sedentary lifestyle. ${ }^{8}$

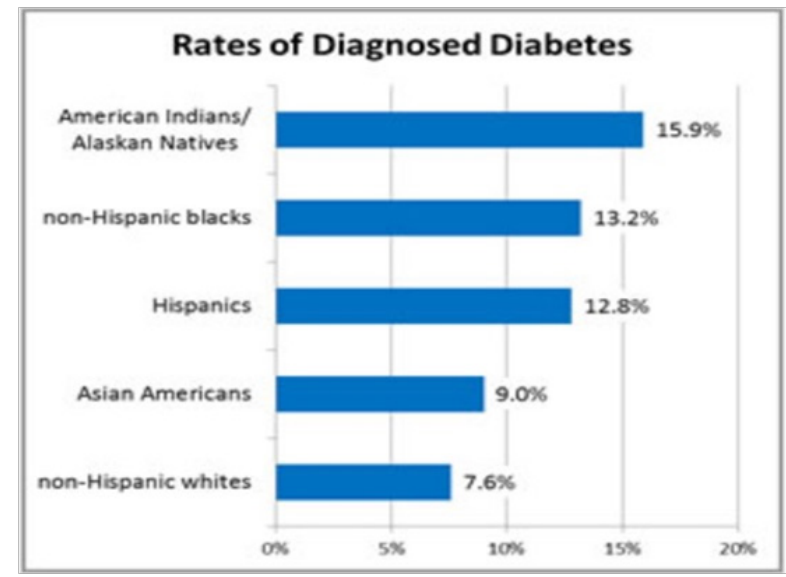

Figure I Statistics about Diabetes.

A study was conducted to determine how geographical location and dietary and life style practices can impact the incidence and prevalence of diabetes. The findings showed that people living in densely populated areas are at a higher risk for diabetes when compared to those who living in rural areas. ${ }^{5}$ The diagnosis and treatment of diabetes have far reaching financial implications that must also be addressed. In 2012 healthcare expenditure on total costs of diagnosed diabetes was an alarming \$245 billion. Another \$176 billion were spent on direct medical costs and $\$ 69$ billion was lost on reduced productivity related to complications of this disease. ${ }^{1}$

\section{Alzheimer's disease}

Studies have indicated that Alzheimer's disease affects approximately $15 \%$ of older Americans, between ages 65 and 74 , (Figure 2). ${ }^{3}$ Also demonstrated in figure 2, 38\% of Americans, age 85 and older are affected by Alzheimer's disease. It is further established, (Figure 3) that there will be a steady annual increase in incidence and prevalence of $\mathrm{AD}$, as older Americans continue to live longer than 65 years..$^{2-5}$ The prevalence in seniors, ages 65 and older were a staggering 5.1 million in 2015. A study using the data from the U.S. Census and the Chicago Health and Aging Project revealed a higher prevalence in women. ${ }^{6,7}$ Approximately two-thirds of individuals with Alzheimer's disease are women; 3.2 million of the 5.1 million individuals living with this disease are women and 1.9 million are men. ${ }^{4}$

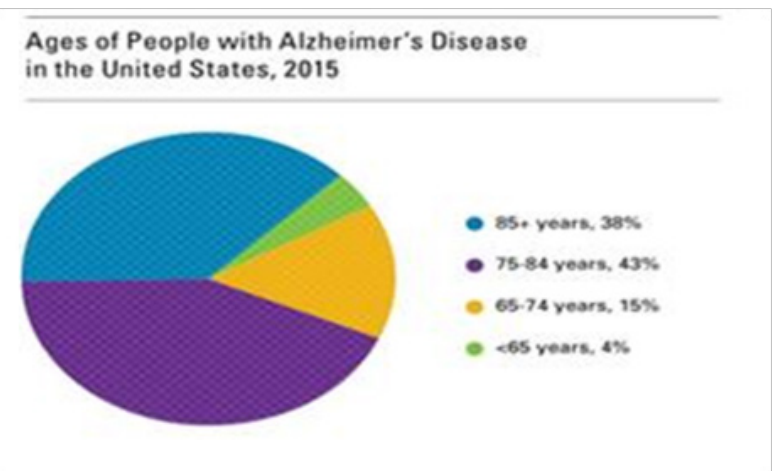

Figure 2 Data from Gaugler et al. ${ }^{2}$

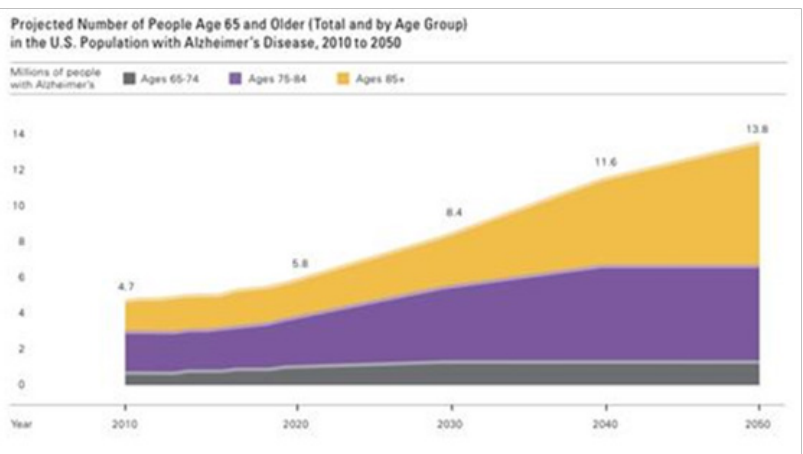

Figure 3 Data from Gaugler et al. ${ }^{2}$

There is data to support that major risk factors for susceptibility to Alzheimer's disease are an individual's age and gender. ${ }^{14}$ In (Figure 4), The Framingham Study inferred that more women than men have the disease. This is because more women live longer than men. Men are more prone to cardiovascular problems compared to women of middle age. ${ }^{2-12}$ As for the men living longer than age 65 , they usually have a lower risk for cardiovascular disease based on their health history and hence, a lower risk for dementia. ${ }^{2-13}$ Since having a higher level of education decreased the likelihood of developing $\mathrm{AD}$, males had a lesser chance of developing AD. This is owing to the fact that men age 65 and older had a higher educational status than women born in the early1900s. ${ }^{14}$ Fewer women acquired higher education in that era and were unlikely to achieve a higher level on education. ${ }^{14}$ Based upon the study's findings, this disparity in prevalence seem to suggest that higher education and brain stimulation decreases the risk of $\mathrm{AD}$.

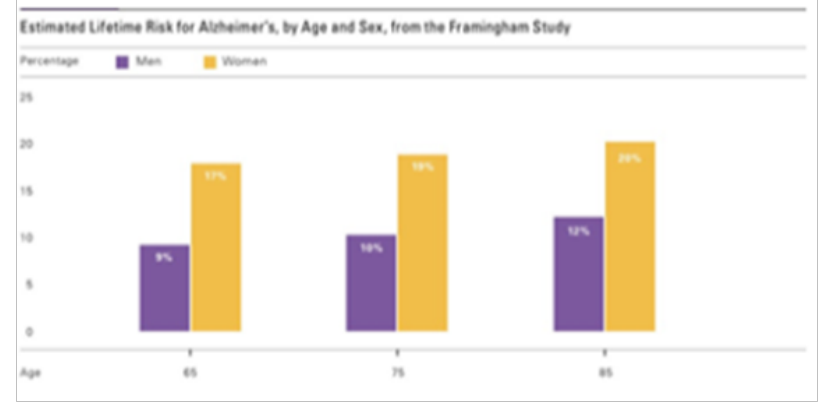

Figure 4 Data from Gaugler et al.2

\section{Linking diabetes and AD}

Several studies have shown that the gene ApoE (Apolipoprotein), specifically the allele E4 ( $\varepsilon 4)$, increases one's risk for developing Alzheimer's disease. This is considered one of the strongest indicators for the detection of $\mathrm{AD} .^{6-13} \mathrm{ApoE}$ is among a group of proteins found in some lipoproteins; for example, Chylomicrons, High-Density lipoproteins (HDLs), Intermediate-Density Lipoproteins (IDLs), and Very-Low-Density lipoproteins (VLDLs). ${ }^{15} \mathrm{ApoE}$ is vital for the transport of lipids throughout the body. Some research data allow that there are noticeable gender biases with respects to the gene ApoE $(\varepsilon 4){ }^{2-13}$ It is interesting to note that there is a higher probability in women diagnosed with Alzheimer's disease compared to their male counterparts..$^{13}$ This is partly a result of the increased amounts of ApoE $(\varepsilon 4)$ in females due to estrogen and the pathology of tau protein..$^{2-17}$ Individuals with (CI), usually have higher levels of fasting insulin. This finding is supportive of the belief that there exists an interrelationship 
between insulin resistance and AD. ${ }^{32}$ In sum, individuals with higher fasting insulin are more prone to develop $\mathrm{AD} .^{7-13}$

The combination of allele $\varepsilon 4$ and glucose intolerance increases the risk factors for $\mathrm{AD}$. However, $\varepsilon 4$ levels did not affect fasting glucose values but it did affect glucose tolerance. ${ }^{7-13}$ In the same studies men had higher fasting glucose than women but women had higher levels of $\varepsilon 4$. This is significant because $\mathrm{ApoE} \varepsilon 4$ considerably altered the risk for $\mathrm{AD}$ in individuals with diabetes. In addition, mutations in the islet amyloid polypeptide (IAPP) gene have been linked to an increased risk for type II Diabetes. ${ }^{38}$ Furthermore, the combination of diabetes and the APOE $\varepsilon 4$ gene exacerbated the predisposing risk factors of $\mathrm{AD}^{6-14}$

\section{Complications of diabetes type II and AD}

As the prevalence of diabetes increases, so too will the incidence of $\mathrm{AD}$ increase; this is because of the interplay of these two diseases. The coexisting complications of diabetes mellitus type II are both pervasive and disheartening. Some of the most common complications linked to diabetes mellitus are kidney failure, ocular impairment, $\mathrm{CI}$, decreased blood flow to the brain, stroke, and cardiovascular complications. ${ }^{16-23}$ These complications are linked to insulin resistance with abnormalities in lipoproteins such as high levels of low-density lipoproteins, low levels of high-density lipoproteins, and high levels of triglycerides. ${ }^{10}$ Fasting hyperglycemia is the result of insulin resistance. This is predicated upon the failure to suppress hepatic gluconeogenesis. Insulin resistance is a result of a dense calorie diet, a sedentary lifestyle, and the usage of some medications like steroids. ${ }^{6}$ When fasting glucose is above the normal range the body is unable to compensate for this and insulin becomes inadequate to return sugar levels to normal. Also, insulin resistance leads to an increase in IAPP production, since IAPP and insulin are cosecreted and subjected to the same regulatory mechanisms. ${ }^{39}$ Thus, in the prediabetes stages, obesity and insulin resistance may increase IAPP production.

Several complications associated with Alzheimer's disease include brain shrinkage, memory loss, loss of inhibitions, depression, incontinence, aphasia, dysphagia and aspiration. There are proteins called beta-amyloids that are found in the lipid membranes of nerve cells, which destroy brain cells and interfere with inter-cellular communication. When these proteins aggregate they are called plaques. ${ }^{24}$ A very important protein called tau is most abundantly located in neurons. Their primary role is to stabilize microtubules in order to properly transport nutrients throughout these microtubules. ${ }^{15}$ When they are unable to play this role, they become tangled and ineffective in transporting nutrients. Due to this ineffective transport system resulting from these twists, brain cells die from lack of vital nutrients and subsequently leading to the aforementioned complications. These are the same plaques and tangles that can be seen in the brain tissues affected with Alzheimer disease. ${ }^{17-24}$

\section{Therapeutic interventions and mechanism of action of drugs}

There are several common interventions known to healthcare professionals that are effective in treating diabetes mellitus type II disease such as lifestyle changes (e.g. increased physical activity and consuming a healthy diet) and pharmacological treatments. Diabetes therapies that enhance insulin secretion, sensitivity, or signaling activity, such as metformin, peroxisome proliferator-activated receptor $\mathrm{g}$ agonists, and GLP-1, have been shown to prevent neurodegeneration in model systems. ${ }^{40-42}$ Furthermore, clinical trials are underway to assess the benefits of intranasal insulin (NCT01767909) and diabetes drugs such as liraglutide (NCT01843075) for the treatment of AD. The mechanism of action of metformin depends on the alterations in cellular energy metabolism (the increased AMP/ATP ratio). ${ }^{25}$ Metformin lowers glucose by inhibiting hepatic gluconeogenesis and opposing the action of glucagon. Metformin-mediated inhibition of mitochondrial complex I results in defective cAMP and protein kinase A regulation of glycogen, lipid metabolism, and sugar. ${ }^{25}$

The prevention of $\mathrm{AD}$ also includes lifestyle changes similar to those of diabetes (increased brain activity, higher education, physical activity, and consuming a healthy diet). Pharmacological treatments for $\mathrm{AD}$ are categorized as cholinesterase inhibitors like Aricept, Razadyne, and Exelon, and memantine like Namenda. The mechanism of action of Cholinesterase inhibitors is to impede the process that breaks down acetylcholine, which is a crucial neurotransmitter. ${ }^{26}$ Memantine is an NMDA ( $N$-methyl-D-aspartate) receptor antagonist that regulates the activity of glutamate, another vital neurotransmitter in the brain, which is crucial in learning and retention. Attachment of glutamate to cell surface called NMDA receptors permits calcium to enter the cell. This process is important for cell signaling, as well as learning and memory. However, in Alzheimer's disease, excess glutamate can be released from injured cells, resulting in continued exposure to calcium. This prolonged exposure to calcium speeds up cellular destruction. Memantine aids in the prevention of this destruction by partially blocking the NMDA receptors. ${ }^{26}$

\section{Collaborative treatment modalities}

There are several forms of non-pharmacological modalities that can both prevent and treat diabetes mellitus type II and AD. With convincing evidence that diabetes type II can be prevented or delayed, strategies to implement the primary prevention of this disease in highrisk subjects are urgently needed. A randomized trial revealed that the dietary intervention that is necessary for the prevention and treatment of diabetes type II consists of decreased intake of refined sugars, saturated fats, simple carbohydrates, and alcohol and an increase intake of complex carbohydrates and high fiber intake like vegetables. ${ }^{27,28}$ These studies suggest that there is a better chance of decreasing the risk of diabetes type II with consistent healthy lifestyles changes as opposed to pharmacological interventions. ${ }^{43-47}$ One study concluded that some of the most effective forms of treatments for $\mathrm{AD}$ are enriched group cognitive training or physical exercise and music, and cranial or dorsal stimulation. ${ }^{29}$ This method of treatment may prove to be costeffective with improved quality of life for both the caregiver and the individual with $\mathrm{AD} .{ }^{29}$ Pharmacotherapies appear to delay the progress of AD. The current limits on the efficacy of medications and the requirement for an array of alternative treatments emphasize the need for more pertinent evaluations of non-pharmacological interventions in $\mathrm{AD} .{ }^{43-47}$ Since the incidence and prevalence of diabetes co-infection with $\mathrm{AD}$ are highest in socioeconomically challenged Blacks and Hispanics, measures at primary prevention must be developed to target these specific populations. ${ }^{1-3}$

\section{Conclusion}

Despite the surplus of research conducted on both diabetes mellitus type II and Alzheimer's disease, further research is needed to prevent and effectively treat both diseases. This allows for sustained circumvention of the debilitating effects and can lead to enhanced quality of life. In light of gender and racial disparities, the most at risk 
populations must be identified and targeted by healthcare educators and public health officials. These individuals must be willing to develop effective educational and clinical tools leading to increased awareness and subsequent disease prevention. Although healthcare professionals and researchers are the main individuals to implement the gold standard of effective methodologies for optimal clinical outcomes, pharmacological treatments have been their preferred intervention. Given that the effects of these diseases are multifaceted; any responsible intervention requires a collaborative approach. In this way, improved clinical outcomes, patient longevity, and overall quality of life can be realized.

\section{Acknowledgements}

None.

\section{Conflict of interest}

The author declares no conflict of interest.

\section{References}

1. Statistics about Diabetes, USA;

2. Gaugler J, James B, Johnson T, et al. 2015 Alzheimer's Disease Facts and Figures. 2015;11(3):p. 1-88.

3. Alzheimer's and Public Health Spotlight: Race Ethnicity and Alzheimer's Disease, USA.

4. Hebert LE, Weuve J, Scherr PA, et al. Alzheimer disease in the United States (2010-2050) estimated using the 2010 Census. Neurology. 2013;80(19):1778-1783.

5. Strachan MW, Reynolds RM, Frier BM, et al. The relationship between type 2 diabetes and dementia. Br Med Bull. 2008;88(1):131-146.

6. Peila R, Rodriguez BL, Launer LJ. Type 2 Diabetes, APOE Gene, and the Risk for Dementia and Related Pathologies: The Honolulu-Asia Aging Study. Diabetes. 2002;51(4):1256-1262.

7. Hanson AJ, Banks WA, Hernandez Saucedo H, et al. Apolipoprotein E Genotype and Sex Influence Glucose Tolerance in Older Adults: A CrossSectional Study. Dement Geriatr Cogn Dis Extra. 2016;6(1):78-89.

8. Edmonds L. Could Alzheimer's be Type 2 diabetes? Scientists claim extra insulin produced by those with disease disrupts brain chemistry. 2013.

9. Sauer A. How Alzheimer's Could Be Type 2 Diabetes. 2016.

10. Khardori R. Type 2 Diabetes Mellitus. 2016.

11. Leading Causes of Death. National Center for Health Statistics. 2016.

12. Chêne G, Beiser A, Au R, et al. Gender and incidence of dementia in the Framingham Heart Study from mid-adult life. Alzheimers Dement. 2014;11(3):310-320.

13. Altmann A, Tian L, Henderson VW, et al. Sex modifies the APOE-related risk of developing Alzheimer disease. Ann Neuro. 2014;75(4):563-573.

14. Sando SB, Melquist S, Cannon A, et al. Risk-reducing effect of education in Alzheimer's disease. Int J Geriatr Psychiatry. 2008;23(11):11561162 .

15. Apolipoprotein E.

16. Moran $\mathrm{C}$, Phan TG, Chen J, et al. Brain atrophy in type 2 diabetes: regional distribution and influence on cognition. Diabetes Care. 2013;36(12):4036-4042.
17. Alzheimer's Brain Tangles-Alzheimer's Association, USA;

18. Biller J, Love BB. Diabetes and stroke. Med Clin North Am. 1993;77(1):95-110.

19. Tuomilehto J, Rastenyte D, Jousilahti P, et al. Diabetes mellitus as a risk factor for death from stroke. Prospective study of the middle-aged Finnish population. Stroke. 1996;27(2):210-215.

20. Danaei G, Lawes CM, Vander Hoorn S, et al. Global and regional mortality from ischemic heart disease and stroke attributable to higher-than-optimum blood glucose concentration: comparative risk assessment. Lancet. 2006;368(9548):1651-1659.

21. Haffner SM, D Agostino R, Mykkänen L, et al. Insulin sensitivity in subjects with type 2 diabetes. Relationship to cardiovascular risk factors: the Insulin Resistance Atherosclerosis Study. Diabetes Care. 1999;22(4):562-568.

22. Busko M. Gray-matter atrophy may drive cognitive decline in diabetes. Medscape Medical News, USA; 2013.

23. Mankovsky BN, Metzger BE, Molitch ME, et al. Cerebrovascular disorders in patients with diabetes mellitus. $J$ Diabetes Complications. 1996;10(4):228 -242.

24. Alzheimer's disease. 2015.

25. Pernicova I, Korbonits M. Metformin-mode of action and clinical implications for diabetes and cancer. Nat Rev Endocrinol. 2014;10(3):143-156.

26. Medication for Memory Loss. Alzheimer's Association, USA; 2017.

27. Tuomilehto J. Nonpharmacologic Therapy and Exercise in the Prevention of Type 2 Diabetes. Diabetes Care. 2009;32(Suppl 2):189-193.

28. Diabetes Prevention Program Research Group. Reduction in the incidence of type 2 diabetes with lifestyle intervention or metformin. $N$ Engl J Med. 2002;346:393-403.

29. Olazarán J, Reisberg B, Clare L, et al. Nonpharmacological Therapies in Alzheimer's Disease: A Systematic Review of Efficacy. Dementia and Geriatric Cognitive Disorders. 2010;30:161-178.

30. Zhong Y, Miao Y, Jia WP, et al. Hyperinsulinemia, insulin resistance and cognitive decline in older cohort. Biomed Environ Sci. 2012;25(1):8-14.

31. Willette AA, Bendlin BB, Starks EJ, et al. Association of insulin resistance with cerebral glucose uptake in late middle-aged adults at risk for Alzheimer's disease. JAMA Neurol. 2015;72(9):1013-1020.

32. Dineley KT, Jahrling JB, Denner L. Insulin resistance and Alzheimer's disease. Neurobiol Dis. 2014;72:92-103.

33. Peripheral hyperinsulinemia and reduced insulin sensitivity in patients with $\mathrm{AD}$ are associated with memory impairment. 2012.

34. Jiménez-Palomares M, Ramos-Rodríguez JJ, López-Acosta $\mathrm{JF}$, et al. Increased $\mathrm{A} \beta$ production prompts the onset of glucose intolerance and insulin resistance. Am J Physiol Endocrinol Meta. 2012;302(11):1373E-1380E.

35. Clarke JR, Lyra E Silva NM, Figueiredo CP, et al. Alzheimer Associated $A \beta$ Oligomers Impact The Central Nervous System To Induce Peripheral Metabolic Deregulation (2015). EMBO Mol Med. 2015;7(2):190-210.

36. Zhang Y, Zhou B, Zhang F, et al. Amyloid- $\beta$ Induces Hepatic Insulin Resistance By Activating JAK2/STAT3/SOCS-1 Signaling Pathway (2012). Diabetes. 2012;61(6):1434-1443.

37. Takeda S, Sato N, Uchio-Yamada K, et al. Diabetes-accelerated Memory Dysfunction via Cerebrovascular Inflammation and $A \beta$ Deposition In An Alzheimer Mouse Model With Diabetes. Proc Natl Acad Sci U S A. 2010;107(15):7036-7041. 
38. Ashraf GM, Greig NH, Khan TA, et al. Protein misfolding and aggregation in Alzheimer's disease and type 2 diabetes mellitus. CNS Neurol Disord Drug Targets. 2014;13(7):1280-1293.

39. Jo W M, Höppener, Bo Ahrén, et al. Islet Amyloid and Type 2 Diabetes Mellitus. N Engl J Med. 2000;343:411-419.

40. Hsu CC, Wahlqvist ML, Lee MS, et al. Incidence of Dementia Is Increased In Type 2 Diabetes and Reduced by The Use Of Sulfonylureas And Metformin. J Alzheimers Dis. 2011;24(3):485-493.

41. Combs CK, Johnson DE, Karlo JC, et al. Inflammatory mechanisms in Alzheimer's disease: inhibition of beta-amyloid-stimulated proinflammatory responses and neurotoxicity by PPARgamma agonists. J Neurosci. 2000;20(2):558-567.

42. McClean PL, Parthsarathy V, Faivre E, et al. The Diabetes Drug Liraglutide Prevents Degenerative Processes In A Mouse Model of Alzheimer's Disease. J Neurosci. 2011;31(17):6587-6594.

43. Eriksson KF, Lindgärde F. Prevention of Type 2 (non-insulin-dependent) Diabetes Mellitus by Diet and Physical Exercise. Diabetologia. 1991;34(12):891-898.
44. Lindgärde F, Eriksson K-F, Lithell H, et al. Coupling Between Dietary Changes, Reduced Body Weight, Muscle Fibre Size and Improved Glucose Tolerance in Middle-aged Men With Impaired Glucose Tolerance. Acta Med Scan. 1982;212:99-106.

45. Saltin B, Lindgärde $F$, Houston $M$, et al. Physical training and glucose tolerance in middle-aged men with chemical diabetes. Diabetes. 1979;28(Suppl 1):30-32.

46. Saltin B, Lindgärde F, Lithell $\mathrm{H}$, et al. Metabolic effects of longterm physical training and in maturity onset diabetes. In: Waldhäusl WK, editor. Diabetes 1979. Proceedings of the $10^{\text {th }}$ Congresss of the International Diabetes Federation. Excerpta Medica, Netherlands; 1980. p. $345-350$.

47. Eriksson KF, Lindgärde F. Intervention strategy in impaired glucose tolerance-the role of physical fitness. In: Smith U, Eriksson S, Lindärde F, editors. Genetic susceptibility to environmental factor-a challenge for public intervention. Almqvist \& Wiksell Internation, Stockholm, Sweden; 1988. p. 75-81. 